\title{
Modeling the excitation of acoustic modes in $\alpha$ Centauri A
}

\author{
R. Samadi ${ }^{1}$, K. Belkacem ${ }^{1}$, M. J. Goupil ${ }^{1}$, M.-A. Dupret ${ }^{1}$, and F. Kupka ${ }^{2}$ \\ 1 Observatoire de Paris, LESIA, CNRS UMR 8109, 92195 Meudon, France \\ e-mail: reza.Samadi@obspm.fr \\ 2 Max-Planck-Institute for Astrophysics, Karl-Schwarzschild Str. 1, 85748 Garching, Germany
}

Received 26 September 2007 / Accepted 30 May 2008

\section{ABSTRACT}

\begin{abstract}
From different seismic observations we infer the energy supplied per unit of time by turbulent convection to the acoustic modes of $\alpha$ Centauri A (HD 128620), a star that is similar but not identical to the Sun. The inferred rates of energy supplied to the modes (i.e. mode excitation rates) are found to be significantly higher than in the Sun. They are compared with those computed with an excitation model that includes two sources of driving, the Reynolds stress contribution and the advection of entropy fluctuations. The model also uses a closure model, the Closure Model with Plumes (CMP hereafter), that takes the asymmetry between the up- and down-flows (i.e. the granules and plumes, respectively) into account. Different prescriptions for the eddy-time correlation function are also compared to observational data. Calculations based on a Gaussian eddy-time correlation underestimate excitation rates compared with the values derived from observations for $\alpha$ Centauri A. On the other hand, calculations based on a Lorentzian eddy-time correlation lie within the observational error bars. This confirms results in the solar case. Compared to the helioseismic data, those obtained for $\alpha$ Centauri A constitute an additional support for our model of excitation. We show that mode masses must be computed taking turbulent pressure into account. Finally, we emphasize the need for more accurate seismic measurements in order to distinguish between the CMP closure model and the quasi-normal approximation in the case of $\alpha$ Centauri A, as well as to confirm or not the need to include the excitation by the entropy fluctuations.
\end{abstract}

Key words. convection - turbulence - stars: oscillations - stars: atmospheres

\section{Introduction}

The star $\alpha$ Centauri A is the most promising after the Sun for constraining the modeling of p-mode excitation by turbulent convection. Indeed, due to its proximity and its binarity, the fundamental parameters of $\alpha$ Centauri A (effective temperature, luminosity, metallicity, gravity, radius) are quite well known. For this reason this star and its companion ( $\alpha$ Cen B) have been extensively studied (see for instance the most recent modeling by Miglio \& Montalbán (2005) and the references therein). As pointed out by Samadi et al. (2007a), the detection of p-modes and the measurement of their amplitudes as well as their mode linewidths (i.e. lifetime), from $\alpha$ Centauri A enable the rates at which energy is supplied to the acoustic modes for this star to be derived. These observational constraints can then be used to check models of p-mode excitation by turbulent convection.

Such comparisons were first undertaken in the case of the Sun by various authors (see the recent review by Houdek 2006). They enable different models of stochastic excitation of acoustic modes to be tested as well as different models of turbulent convection (see e.g. Samadi et al. 2006). Among those theoretical prescriptions, we consider that one of Samadi \& Goupil (2001) with the improvements proposed by Samadi et al. (2003) and Belkacem et al. (2006b). It was shown by Samadi et al. (2003) that the way the eddy time correlation is modeled plays an important role in the efficiency of excitation. Indeed, calculations of the mode excitation rates, $\mathcal{P}$, that use a Lorentzian eddy-time correlation function reproduce helioseismic data better than those using a Gaussian one. In addition, Belkacem et al. (2006b), in the case of the Sun, show that excitation rates computed using an adapted closure model that takes the presence of plumes into account reproduce the solar observations much better than the calculations based on the classical quasi-normal approximation (Millionshchikov 1941).

An alternative theoretical model of the excitation of acoustic modes by turbulent convection proposed by Chaplin et al. (2005) differs from that by Samadi \& Goupil (2001) in several ways: it does not take the driving by the advection of the entropy fluctuations by the velocity field into account. They only use the classical quasi-normal approximation. More importantly, these authors claim that a Gaussian eddy-time correlation function reproduces one the frequency dependence of mode excitation rates inferred from helioseismic data better than a Lorentzian one. However, they are led to introduce a factor to their model by which they multiply their formulation to reproduce the maximum of the solar mode excitation rates.

A second opportunity is provided by $\alpha$ Centauri A for testing various assumptions in the modeling of the p-mode excitation: the amplitudes of the acoustic modes detected in $\alpha$ Centauri A were derived by Butler et al. (2004) using spectrometric data. From those data, an estimate of the averaged mode linewidths has been first proposed by Bedding et al. (2004) and more recently updated in Kjeldsen et al. (2005). Using a different method and data from the WIRE satellite, Fletcher et al. (2006) propose a new estimate of the averaged mode linewidths that differ significantly from the one derived by Kjeldsen et al. (2005). Indeed, the two data sets place the mode lifetime between 2.2 days (Kjeldsen et al. 2005) and 3.9 days (Fletcher et al. 2006). For comparison, the averaged mode life time derived for the Sun by Bedding et al. (2004) in a similar way as for $\alpha$ Centauri A by Kjeldsen et al. (2005) is about two days. 
Samadi et al. (2007a) inferred the p-mode excitation rates $\mathcal{P}$ from those sets of seismic constraints. They find that they are significantly larger than those associated with the solar p-modes. Furthermore, $\mathcal{P}$ peaks in the frequency domain $\sim 2.2-2.6 \mathrm{mHz}$, while it peaks at the frequency $v_{\max } \sim 3.8 \mathrm{mHz}$ in the case of the Sun.

Although the spectroscopic characteristics $\left(T_{\mathrm{eff}}=5810 \mathrm{~K}\right.$, $\log g=4.305)$ of $\alpha$ Centauri A are close to those of the Sun $\left(T_{\text {eff }}=5780 \mathrm{~K}, \log g=4.438\right)$, the seismic signatures are quite different. Consequently, finding agreement between predicted and observed excitation rates would be a nontrivial result, providing additional support for the theory.

A preliminary comparison with theoretical calculations obtained in the manner of Belkacem et al. (2006a) was carried out by Samadi et al. (2007a). Discrepancies between the excitation rates inferred from the observations and the theoretical calculations were found. We update this study here by proceeding in a similar way as in Rosenthal et al. (1999). Indeed, these authors have built a solar 1D model where the surface layers are taken directly from a fully compressible 3D hydrodynamical numerical model. We refer here to such a 1D model as a "patched" model. Rosenthal et al. (1999) have obtained a much better agreement between observed and theoretical eigenfrequencies of the Sun computed for such a "patched" 1D model than for a "standard" 1D model based on the standard mixing-length theory with no turbulent pressure included. Following Rosenthal et al. (1999), we built such a "patched" model to derive adiabatic mode radial eigen-displacements $\left(\xi_{r}\right)$ and mode inertia $(I)$. We used them to compute the mode excitation rates, which we compared with excitation rates computed using $\xi_{r}$ and $I$ obtained with a "standard" $1 \mathrm{D}$ model.

The paper is organized as follows: in Sect. 2 we describe our procedure for computing the mode excitation rates for the specific case of $\alpha$ Centauri A. We then describe in Sect. 3 the way the mode excitation rates are inferred from seismic observations of $\alpha$ Centauri A. In Sect. 4, we compare theoretical calculations of $\mathcal{P}$ with those inferred from the seismic data obtained for $\alpha$ Centauri A. We compare and explain in Sect. 5 the differences between $\alpha$ Centauri A and the Sun. Finally, Sects. 6 and 7 are devoted to the discussion and conclusions, respectively.

\section{Modeling the excitation of p-modes}

\subsection{General formulation}

Because the theoretical model of stochastic excitation is basically that of Samadi \& Goupil (2001; see also Samadi et al. 2005) with the improvements of Belkacem et al. (2006a,b), we recall only some key features here. The model takes two driving sources into account. The first one is related to the Reynolds stress tensor and, as such, represents a mechanical source of excitation. The second one is caused by the advection of the turbulent fluctuations of entropy by the turbulent motions (the so-called "entropy source term") and thus represents a thermal source of excitation (Goldreich et al. 1994; Stein \& Nordlund 2001). The power fed into each radial mode, $\mathcal{P}$, is given by

$\mathcal{P}=\frac{1}{8 I}\left(C_{\mathrm{R}}^{2}+C_{\mathrm{S}}^{2}\right)$,

where $C_{\mathrm{R}}^{2}$ and $C_{\mathrm{S}}^{2}$ are the turbulent Reynolds stress and entropy contributions, respectively and

$I=\int_{0}^{M} \mathrm{~d} m\left|\xi_{r}\right|^{2}$ is the mode inertia, $\xi_{r}$ is the adiabatic radial mode displacement and $M$ the mass of the star. The expressions for $C_{\mathrm{R}}^{2}$ and $C_{\mathrm{S}}^{2}$ are given for a radial mode with frequency $\omega_{0}$ by

$$
\begin{aligned}
& C_{\mathrm{R}}^{2}=\frac{64 \pi^{3}}{15} \int \mathrm{dm} \rho_{0} f_{r}\left(1+\frac{1}{3} \mathcal{S}_{w}^{2}\right) S_{\mathrm{R}}\left(\omega_{0}\right) \\
& C_{\mathrm{S}}^{2}=\frac{16 \pi^{3}}{3 \omega_{0}^{2}} \int \mathrm{dm} \frac{\alpha_{s}^{2}}{\rho_{0}} g_{r} S_{\mathrm{S}}\left(\omega_{0}\right)
\end{aligned}
$$

where we have defined

$$
\begin{aligned}
& S_{\mathrm{R}}\left(\omega_{0}\right)=\int \frac{\mathrm{d} k}{k^{2}} E^{2}(k) \int \mathrm{d} \omega \chi_{k}\left(\omega+\omega_{0}\right) \chi_{k}(\omega), \\
& S_{\mathrm{S}}\left(\omega_{0}\right)=\int \frac{\mathrm{d} k}{k^{2}} E(k) E_{s}(k) \int \mathrm{d} \omega \chi_{k}\left(\omega+\omega_{0}\right) \chi_{k}(\omega),
\end{aligned}
$$

where $f_{r} \equiv\left(\mathrm{d} \xi_{r} / \mathrm{d} r\right)^{2}$, and $g_{r}$ is a function that involves the first and the second derivatives of $\xi_{r}$. Then, $E(k)$ is the spatial turbulent kinetic energy spectrum, $E_{s}(k)$ the spectrum associated with the entropy fluctuations, $\chi_{k}$ the time correlation function of the eddies, $\alpha_{s}=(\partial P / \partial s)_{\rho}$ where $s$ is the entropy, $P$ the gas pressure, $\rho$ the density, $\rho_{0}$ the equilibrium density profile, and $\omega_{0}$ the eigenfrequency.

Finally, $\mathcal{S}_{w} \equiv\left\langle w^{3}\right\rangle /\left(\left\langle w^{2}\right\rangle\right)^{3 / 2}$ is the skewness and $w$ the vertical component of the velocity (see Belkacem et al. 2006a,b, for details). Indeed, the expression of Eq. (3) depends on the closure model used to express the fourth-order moments involved in the theory in terms of the second-order ones. The most commonly used closure model at the level of fourth-order moments is the quasi-normal approximation (QNA). Such an assumption leads to a vanishing skewness $\mathcal{S}_{w}$. However, in the solar case, the deviation from the QNA stems from the presence of turbulent plumes. Taking both the effect of the skewness introduced by the presence of two flows and the effect of turbulence onto each flow into account Belkacem et al. (2006a) thus propose a new closure model, which leads to a non-vanishing skewness, $\mathcal{S}_{w}$, in Eq. (3). In the present work, $\mathcal{S}_{w}$ is then obtained directly from the 3D numerical model.

Calculation of the mode excitation rates is performed essentially in the manner of Belkacem et al. (2006b) as explained in Samadi et al. (2007a) in the specific case of $\alpha$ Centauri A: all required quantities, except the mode eigenfunctions $\xi_{r}$ and mode inertia $I$, are directly obtained from a $3 \mathrm{D}$ simulation of the outer layers of $\alpha$ Centauri A, whose characteristics are described in Sect. 2.2 below.

The mode displacement $\xi_{r}$ and mode inertia $I$ must be computed from a global 1D equilibrium model. We chose to study two such equilibrium models which are described in Sect. 2.3. Finally, eigenfrequencies and eigenfunctions are computed using the adiabatic pulsation code ADIPLS (Christensen-Dalsgaard \& Berthomieu 1991).

\subsection{The $3 D$ hydrodynamical model of the outer layers of $\alpha$ Centauri $A$}

We consider the 3D hydrodynamical model of the outer layers of $\alpha$ Centauri A computed by Samadi et al. (2007a) using the Stein \& Nordlund (1998) code. The assumed micro-physics (e.g. the equation of state and the opacity table) are explained in Samadi et al. (2007b). The hydrogen, helium, and metal abundances are solar, and the chemical mixture of the heavy elements is set according to the Grevesse \& Noels (1993) mixture.

The 3D model associated with $\alpha$ Centauri A has a horizontal size of $8.17 \mathrm{Mm} \times 8.17 \mathrm{Mm}$ and a vertical size of 4.31 Mm. The 
grid is $125 \times 125 \times 82$. As pointed out by Samadi et al. (2007b), this spatial resolution is sufficient for calculating the p-mode excitation rates. The simulation duration is $323 \mathrm{~min}$, while the acoustic depth of the simulation is $410 \mathrm{~s}$ and the characteristic eddy turnover time is $\sim 20 \mathrm{~min}$ (see Sect. 5). The duration of the simulation then represents $\sim 47$ sound travels across the simulated domain and about 15 eddy turnover times.

The effective temperature $T_{\text {eff }}$ is adjusted to $5809 \mathrm{~K} \pm 15$, in good agreement with the value $T_{\text {eff }}=5810 \mathrm{~K} \pm 50$ adopted by Miglio \& Montalbán (2005). The gravity is set to $\log g=4.305$ to exactly match the value $(\log g=4.305 \pm 0.005)$ inferred from the precise measurements of the mass and the radius of the star (see the related references in Miglio \& Montalbán 2005).

\section{3. $1 D$ models}

\subsubsection{Standard model}

The first $1 \mathrm{D}$ equilibrium model has the effective temperature and the gravity of $\alpha$ Centauri A and is built by requiring that, for the temperature at the bottom of the $3 \mathrm{D}$ simulation box, the 1D model has the same pressure and density as the 3D simulation (see Fig. 1). The 3D simulation is therefore used to constrain this 1D equilibrium model such that its interior structure is compatible with the second 1D model described later on, in Sect. 2.3.2 (see also Fig. 1). Convection in the 1D model is described according to Böhm-Vitense (1958)'s mixing-length local theory of convection (MLT) and turbulent pressure is ignored. Microscopic diffusion of helium and heavy elements are treated according to the simplified formalism of Michaud \& Proffitt (1993). We assume a solar abundance to be consistent with the 3D model.

The mixing-length parameter, $\alpha$, the age, the mass $(M)$, the initial helium abundance $\left(Y_{0}\right)$, and the initial $(Z / X)_{0}$ ratio where $X$ and $Z$ are the hydrogen and metal mass fractions, respectively, are fitted such that the model simultaneously reproduces the effective temperature of the star, its gravity, the solar composition, and the temperature-pressure relation at the bottom of the 3D simulation box. The outer layers of this model, which matter here, then have the stratification given by a standard MLT model. This model is referred to as standard hereafter.

The matching results in $\alpha=1.694$. For comparison, the same matching performed for a solar 3D simulation results in $\alpha=1.899$. The mass of the standard model is $M=1.012 M_{\odot}$ and the radius $R=1.1722 R_{\odot}$. They are slightly less than expected for this star, namely $M=1.105 \pm 0.007 M_{\odot}$ and $R=1.224 \pm 0.003 R_{\odot}$ (see Miglio \& Montalbán 2005). This is because we have assumed a solar abundance for consistency with the 3D model. A global 1D model with the iron-to-hydrogen abundance $([\mathrm{Fe} / \mathrm{H}])$ of $\alpha$ Centauri A (namely $[\mathrm{Fe} / \mathrm{H}]=0.2$ ), would have the expected mass and radius of the star.

Slightly different $R, M$ and $[\mathrm{Fe} / \mathrm{H}]$ values might have some influence on the mode excitation rates $(\mathcal{P})$. To measure the effect of having an $R, M$ and $[\mathrm{Fe} / \mathrm{H}]$ different than required for $\alpha$ Centauri A, we computed two global models. The first model has an abundance $[\mathrm{Fe} / \mathrm{H}]=0.2$ and the second has a solar abundance. Both models have the effective temperature and gravity of $\alpha$ Centauri A. In contrast with the "standard" model described above, we do not match these models with the $3 \mathrm{D}$ model. The model with $[\mathrm{Fe} / \mathrm{H}]=0.2$ almost has the radius and the mass expected for $\alpha$ Centauri A, while the second has almost the same $R$ and $M$ as the standard model investigated here. We find that $\mathcal{P}$ changes between the two models by less than $\sim 5 \%$; this is much less than the uncertainties associated with the observations.

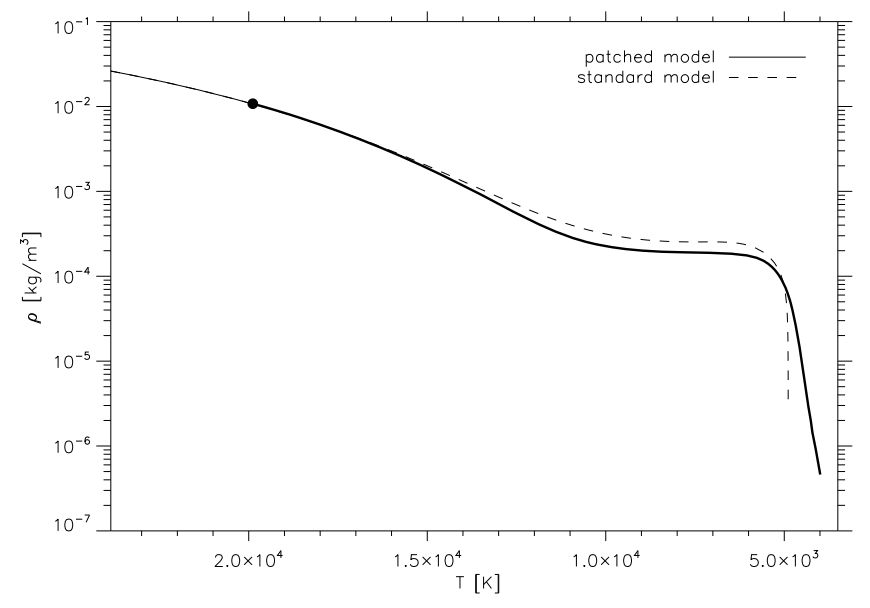

Fig. 1. Density as a function of the temperature. The solid line corresponds to the "patched" model and the dashed line to the "standard" model. The thick solid line is the part of the patched model obtained from the $3 \mathrm{D}$ simulation. The filled circle shows the position of the bottom of the 3D simulation box.

\subsection{2. "Patched" model}

To consider a more realistic description of the superadiabatic outer layers, we built a 1D global model, following Trampedach (1997, see also Samadi et al. 2007b), in which the outer layers are replaced by the averaged 3D simulation (see Fig. 1).

"Standard" and 3D models share the same microphysics but mainly differ in the way convective motions and radiative transfer are treated. In the 3D model convective motions are treated by solving the Navier-Sokes equation while in the standard model convective motions are modeled according to the mixing-length model of convection and no turbulent pressure is included in the hydrostatic equation. In the standard model, radiative transfer is gray and assumes the Eddington approximation. In the 3D model, radiative transfer is explicitly solved in LTE for four opacity bins (see details in Stein \& Nordlund 1998).

The interior of the "patched" model is the same as in the standard model and does not include the turbulent pressure. At the bottom of the $3 \mathrm{D}$ simulation box, turbulent pressure is already negligible ( $\sim 0.6 \%$ of the total pressure). Then, neglecting it in the interior has negligible effects on the properties of the eigenfunctions considered here. This global model will be referred to as a patched model. Note that this patched model has the same total mass and a radius very close to that of the standard model, namely $R=1.1726 R_{\odot}$.

The stratifications in density of the patched and standard models are compared in Fig. 1. At the top of the convective region, we see that the density is lower in the patched model compared to the standard model. This is because the patched model includes turbulent pressure that provides additional support against gravity: accordingly, the patched model has lower gas pressure at a given total pressure $\left(P_{\text {tot }}\right)$. Now, since $T\left(P_{\text {tot }}\right)$ is the same with and without turbulent pressure, the patched model has a lower density at a given temperature than the standard model.

Because the treatment of photospheric radiative transfer is not (and in practice cannot be) identical between a 3D calculation and a 1D model in the atmosphere, small differences in the stratifications of the very outer layers exist between the two models as seen in Fig. 1. In any case these differences do not play any significant role in the quantities that matter here, such as inertia. Note that some explanations about the differences seen 
between the outer layers of 3D models and 1D models have been proposed by e.g. Nordlund \& Stein (1999) and Rosenthal et al. (1999).

\section{Inferring the excitation rates from seismic constraints}

Mode excitation rates are derived from seismic observations according to the relation

$\mathcal{P}(v)=2 \pi \mathcal{M} \Gamma\left(v / S_{0}\right)^{2}$

where $\mathcal{M}=I / \xi_{r}^{2}\left(r_{h}\right)$ is the mode mass evaluated at the layer $r_{h} \equiv R+h$ in the atmosphere where the mode is measured in radial velocity, $R$ the radius at the photosphere (i.e. at $T=T_{\text {eff }}$ ), $h$ the height above the photosphere, $\Gamma$ the mode full width at half maximum (in $v), v\left(r_{h}, v\right)$ is the rms apparent velocity amplitude of the mode at the layer $r_{h}, v=\omega_{0} / 2 \pi$ the mode frequency, and $S_{0}$ the visibility factor of the $\ell=0$ mode.

Kjeldsen et al. (2005) have derived the apparent amplitude velocity spectrum, $v(v)$ of the modes detected in $\alpha$ Centauri A. However, their spectrum corresponds to amplitudes normalized to the mean of $\ell=0$ and 1 modes rather than to $\ell=0$. Furthermore, they do not take the mode visibilities into account. Recently, Kjeldsen et al. (2008) have derived the (apparent) amplitudes of the modes, normalized to the $\ell=0$ modes and taking both the mode visibilities and limb-darkening effects into account. Finally, to derive the intrinsic mode amplitudes, we divide $v(v)$ by $S_{0}=0.712$, the visibility factor of the $\ell=0$ modes observed in velocity (Kjeldsen et al. 2008). For the mode linewidth, $\Gamma$, we use the averaged values provided by Kjeldsen et al. (2005) and Fletcher et al. (2006).

Concerning mode masses, $\mathcal{M}$, as discussed in Sect. 6, it is not trivial to determine the height $h$ where the Doppler velocities are predominantly measured. As we do not know the height representative of the observations precisely, we evaluate the mode masses - by default - at the optical depth $\tau_{500 \mathrm{~nm}} \simeq 0.013$, which corresponds to the depth where the potassium $(\mathrm{K})$ spectral line is formed (but see Sect. 6 for a discussion). This optical depth corresponds to $h=470 \mathrm{~km}$.

Neither the standard nor the patched models have exactly the radius and the mass expected for $\alpha$ Centauri A (see Sect. 2.3.1). However, this inconsistency only has a negligible effect on the mode mass $\mathcal{M}=I / \xi_{r}^{2}$. Indeed, since the eigenmode displacement, $\xi_{r}$, is directly proportional to $R$, the mode inertia $I$ scales as $R^{2}$ (see Eq. (2)). Accordingly, the ratio $I / \xi_{r}^{2}$ is almost insensitive to a small change in $R$. Furthermore, we checked that $\mathcal{M}$ is also insensitive to a small change in $M$.

\section{Comparison between observations and modeling}

We first compare theoretical calculations of $\mathcal{P}$ performed with eigenfunctions computed with the patched equilibrium model with those computed using the standard equilibrium model (see Sect. 2.2). However, eigenmodes computed with those two models do not have the same inertia and hence not the same mode masses $\mathcal{M}$. Thus, we instead compare the ratios $\mathcal{P} / \mathcal{M}$. As shown in Fig. 2, theoretical calculations that use the patched model lie well inside the observed domain of the seismic constraints. On the other hand, using the standard model leads to underestimated theoretical values compared to the two sets of seismic constraints.

When comparing the integrands of the product $\mathcal{P} \mathcal{M}$ - excitation power times mode mass - between the patched and standard

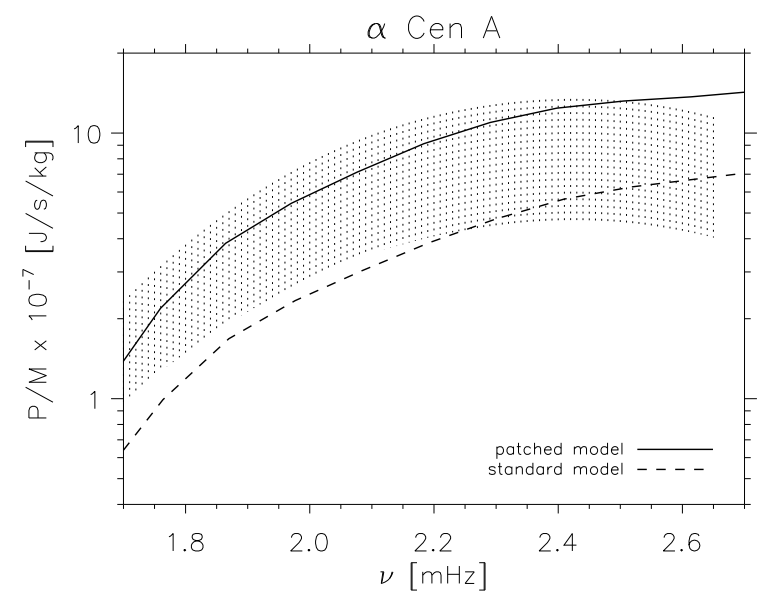

Fig. 2. Ratio of the rates $\mathcal{P}$ at which energy is injected into p-modes to mode masses $(\mathcal{M})$ for $\alpha$ Centauri A. The dashed area represents the observed domain for $\mathcal{P} / \mathcal{M}=2 \pi \Gamma\left(v / S_{0}\right)^{2}$ as a function of $v$. This domain is defined by merging the uncertainties associated with two independently derived values of $\Gamma$ and with the mode amplitudes $v$ (Eq. (7)). The solid (resp. dashed) line corresponds to computed excitation rates with the eigenmodes obtained using the "patched" (resp. "standard") 1D global model. All calculations here use the CMP and the Lorentzian function (LF) for the eddy time-correlation function $\chi_{k}$ in Eqs. (5) and (6).

equilibrium models, we find that they are quite similar. On the other hand, the mode masses $\mathcal{M}$ are quite different for the two equilibrium models in the domain $1-3 \mathrm{mHz}$ where the modes are mostly excited. This is due to the turbulent pressure that is present in the patched model and ignored in the standard model. At a given radius in the super-adiabatic region, the patched model has a lower gas pressure and density (see Fig. 1). As a consequence, inertia of the modes, which are confined within the super-adiabatic region where the turbulent pressure has its maximum, are less for the patched model than for the standard model; accordingly, the ratios $\mathcal{P} / \mathcal{M}$, which are inversely proportional to the squared mode mass $\mathcal{M}^{2}$, are about two times higher for the patched model.

In Fig. 3, we compare two sets of calculations for a patched stellar model that assumes two different prescriptions for the eddy-time correlation function $\left(\chi_{k}\right)$ and two different closure models, namely the QNA and the Closure Model with Plumes (CMP hereafter) in the excitation model. The theoretical calculations based on a Lorentzian $\chi_{k}$ and the CMP closure model lie inside the range allowed by the two sets of seismic constraints. The differences between calculations based on the CMP and on the QNA are found smaller than the differences between the two data sets. On the other hand, calculations based on a Gaussian $\chi_{k}$ yields significantly underestimated values compared to the seismic constraints.

Note that Samadi et al. (2007a) found a discrepancy between theoretical calculations and observations. Part of this discrepancy was due to the horizontal size of the simulation box being set to that of the solar simulation used in Belkacem et al. (2006b). Indeed, the kinetic energy spectrum $E$ involved in the expression for $S_{\mathrm{R}}$ and $S_{\mathrm{S}}$ in Eqs. (5) and (6) must be normalized with respect to the horizontal size of the simulation box, as done here. Furthermore, the mode inertia considered by Samadi et al. (2007a) were computed for a standard MLT model (i.e. no turbulent pressure included) instead of using a patched model as is done here. As shown in Fig. 2, this results in an underestimation of the ratio $\mathcal{P} / \mathcal{M}$ by a factor of about two (see Fig. 2). 


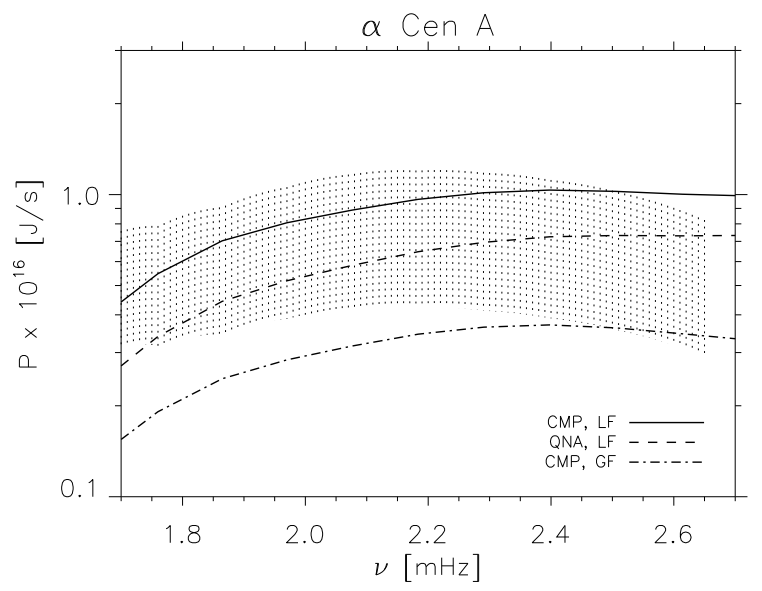

Fig. 3. Rates $\mathcal{P}$ at which energy is injected into the p-modes of $\alpha$ Centauri A. The dashed area has the same meaning as in Fig. 2. The lines correspond to different theoretical calculations (all using a patched model): the solid line uses the Lorentzian function (LF) and the CMP, the dashed line uses the LF and the QNA closure model, the dot-dashed line uses the Gaussian function (GF) and the CMP.

\section{Differences between $\alpha$ Centauri A and the Sun}

\subsection{Excitation rates}

Figure 4 compares the excitation rates, $\mathcal{P}$, inferred for $\alpha$ Centauri A with those inferred from helioseismic measurements obtained for the Sun. For $\alpha$ Centauri A, excitation rates are obtained from the seismic measurements as explained in Sect. 3.

For the Sun we consider the helioseismic data studied by Baudin et al. (2005). We use here solar mode masses obtained with a patched model computed as for $\alpha$ Centauri A in Sect. 2 . Mode masses are evaluated for the optical depth $\tau \simeq 5 \times 10^{-4}$ since SOHO/GOLF observations are based on the Na D1 and D2 spectral lines (see Houdek 2006).

We find $\mathcal{P}_{\max , \odot} \simeq 3.5 \pm 0.4 \times 10^{15}[\mathrm{~J} / \mathrm{s}]$. The excitation rates inferred for $\alpha$ Centauri A with mode masses $\mathcal{M}$ evaluated at the optical depth associated with the potassium line $(\tau \simeq 0.013)$ give $\mathcal{P}_{\max }=8.25 \pm 1.0 \times 10^{15}[\mathrm{~J} / \mathrm{s}]$. This is about $2.3 \pm 0.3$ times larger

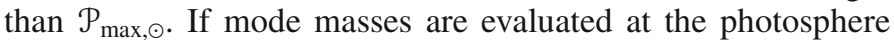
$\left(h=0, T=T_{\text {eff }}\right)$, we obtain $\mathcal{P}_{\max }=15.9 \pm 8.0 \times 10^{15}[\mathrm{~J} / \mathrm{s}]$. In that case this is about $\sim 4.4 \pm 2$ times larger than $\mathcal{P}_{\max , \odot}$.

As seen in Fig. 4, the frequency where $\mathcal{P}$ peaks is $\sim 2.4 \mathrm{mHz}$ for $\alpha$ Centauri A. For comparison, in the solar case, $\mathcal{P}$ peaks around $3.8 \mathrm{mHz}$. Clearly, the modes in $\alpha$ Centauri A are excited at a lower frequency compared to the solar modes. Note also that the frequency domain where the derivation of $\mathcal{P}$ is possible from the available seismic data is much smaller for $\alpha$ Centauri A than for the Sun. This is obviously because the quality of the seismic data is much lower for $\alpha$ Centauri A than for the Sun.

\subsection{Excitation rates as a function of depth}

Figure 5 shows the integrand $\mathrm{d} \mathcal{P} / \mathrm{d} m$ of the excitation rates (Eq. (1), (3), (4)) as a function of the temperature for the mode for which $\mathcal{P}$ is maximum in the Sun and in $\alpha$ Centauri A. The top panel shows the contribution of the Reynolds stress $\left(\mathrm{d} \mathcal{P}_{\mathrm{R}} / \mathrm{d} m\right)$ and the bottom panel the contribution of the entropy fluctuations $\left(\mathrm{d} \mathcal{P}_{\mathrm{S}} / \mathrm{d} m\right)$. Excitation due to the Reynolds stress is maximum where the rms value of velocity, $u$, peaks. Excitation due to the entropy fluctuations is maximum where $\tilde{s}$, the rms value of the entropy fluctuations, peaks. Figure 5 shows that the excitation

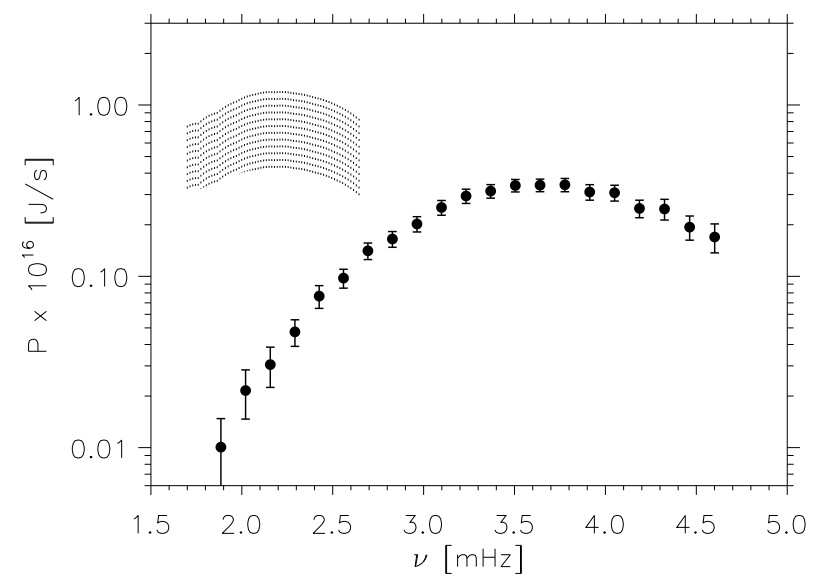

Fig. 4. Excitation rates $\mathcal{P}$ inferred from seismic data according to Eq. (7). Filled circles correspond to the helioseismic constraints obtained by Baudin et al. (2005). The dashed area represents the observed domain for the excitation rates derived for $\alpha$ Centauri A.

rate is larger for $\alpha$ Centauri A than for the Sun and occurs over a slightly more extended region in $\alpha$ Centauri A than in the Sun.

The excitation due to the entropy fluctuations occurs in a more shallow region compared to the Reynolds stress. For $\alpha$ Centauri A, the relative contribution of the entropy fluctuations to the total excitation is $\simeq 18 \%$, which is similar to the Sun $(\simeq 15 \%)$. Hence, in both cases the excitation due to the Reynolds stress remains the dominant contribution.

\subsection{Differences in the characteristic properties of convection}

To summarize, we find that $\mathcal{P}$ is significantly larger in $\alpha$ Centauri A than in the Sun. Furthermore, $\mathcal{P}$ peaks at lower frequency. As shown below, all these seismic differences can be attributed to differences in the characteristic properties of convection between $\alpha$ Centauri A and the Sun.

\subsubsection{Why $\mathcal{P}$ is larger for $\alpha$ Centauri $\mathrm{A}$ ?}

At a given layer, the power supplied to the modes by the Reynolds stress is proportional - per unit mass - to $\rho_{0} u^{3} \Lambda^{4}$ where $\Lambda$ is the characteristic eddy size and $u$ the rms value of the velocity (see Samadi \& Goupil 2001). The flux of the kinetic energy, $F_{\text {kin }}$, is proportional to $\rho_{0} u^{3}$. Hence, the greater $F_{\text {kin }}$ or $\Lambda$, the greater the driving by the Reynolds stress.

The power supplied to the modes by the so-called entropy source term is proportional - per unit mass to $\rho_{0} u^{3} \Lambda^{4} \mathcal{R}^{2} /\left(\tau_{\Lambda} \omega_{0}\right)^{2}$ where $\omega_{0}$ is the mode frequency, $\tau_{\Lambda} \sim \Lambda / u$ is the characteristic eddy turn over time, and finally $\mathcal{R} \propto F_{\text {conv }} / F_{\text {kin }}$ where $F_{\text {conv }} \propto w \alpha_{s} \tilde{s}$ is the convective flux and $\tilde{s}$ is the rms of entropy fluctuations (see Samadi et al. 2006). The higher $\mathcal{R}$, the higher the relative contribution of the entropy source to the excitation. The driving is maximum for mode frequency (see, e.g., Samadi \& Goupil 2001)

$\omega_{0} \sim 2 \pi / \tau_{\Lambda}$.

Hence, at the mode frequency $\omega_{0} \sim 2 \pi / \tau_{\Lambda}$, the higher the ratio $F_{\text {conv }} / F_{\text {kin }}$, the greater the relative contribution of the entropy source term to the total excitation rate.

As a summary, for both Reynolds stress and entropy contributions, the larger the characteristic scale length $(\Lambda)$ or the higher the kinetic energy $\left(F_{\text {kin }}\right)$, the greater the excitation. Furthermore, the higher $\mathcal{R}$, the greater the relative contribution 

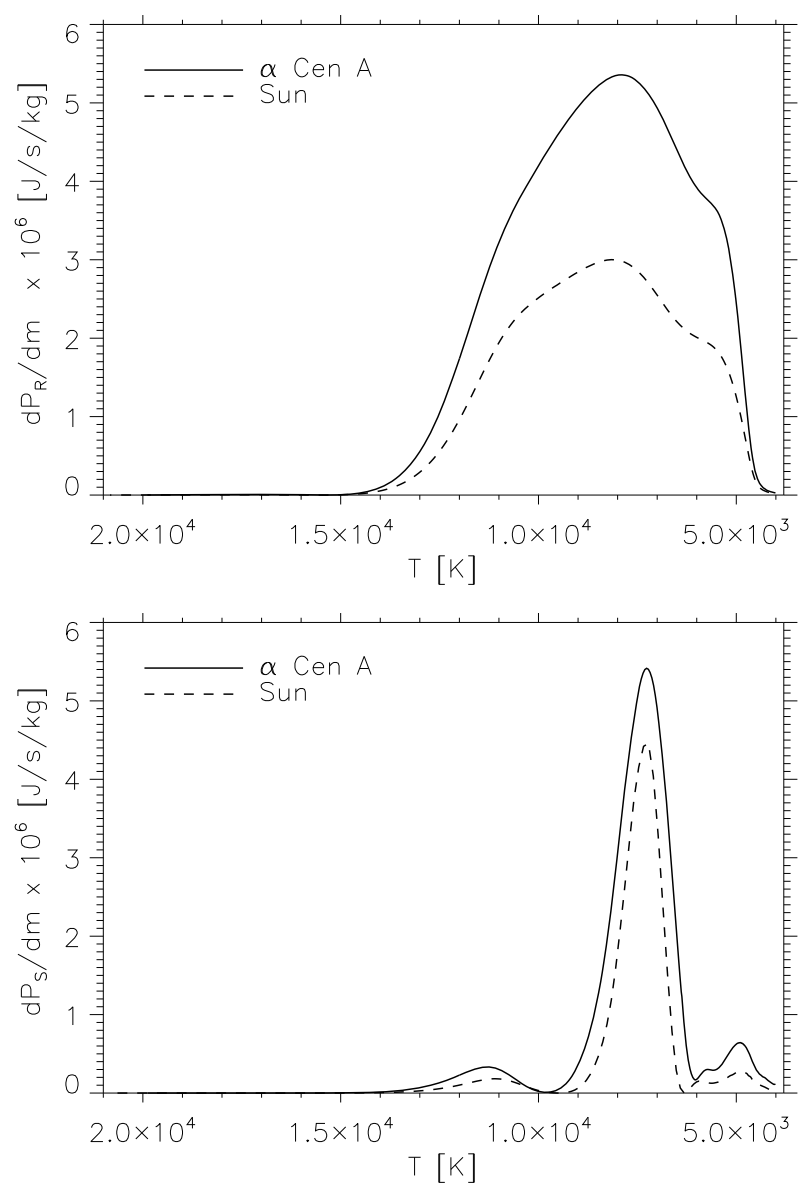

Fig. 5. Top: the integrand $\mathrm{d} \mathcal{P}_{\mathrm{R}} / \mathrm{d} m$ (Eq. (1)) associated with the contribution of the Reynolds stress to the excitation is plotted as a function of the horizontally and temporally averaged temperature in the simulation box for the mode for which $\mathcal{P}$ is maximum. The solid line corresponds to the 3D simulation associated with $\alpha$ Centauri A and the dashed line to the one associated with the Sun. Bottom: as the top panel for $\mathrm{d} \mathcal{P}_{\mathrm{S}} / \mathrm{d} m$, the integrand associated with the contribution due to the entropy fluctuations.

of the entropy source term to the excitation. We study the differences in $\Lambda, F_{\text {kin }}$, and $\mathcal{R}$ between the Sun and $\alpha$ Centauri A below.

Kinetic energy flux $\left(F_{\text {kin }}\right)$ :

The maximum in $u$ is up to $\sim 10 \%$ greater in the $3 \mathrm{D}$ simulation associated with $\alpha$ Centauri A than in the solar one. However, the differences in the flux of kinetic energy, $F_{\text {kin }}$, between the 3D simulations associated with $\alpha$ Centauri A and the solar one are small $(\$ 10 \%)$. This small effect on $F_{\text {kin }}$ despite its cubic dependence on $u$ is due the lower $\rho_{0}$ for a layer with the same average $T$ in the simulation for $\alpha$ Centauri A as compared to the simulation for the Sun. The lower $\rho_{0}$ in turn is a consequence of the lower surface gravity of $\alpha$ Cen A compared to the Sun.

Relative contribution of the entropy source term:

We also find that $\tilde{s}$ is $\sim 25 \%$ greater in the $3 \mathrm{D}$ simulation associated with $\alpha$ Centauri A. However, the convective flux, $F_{\text {conv }} \propto$ $w \alpha_{s} \tilde{s}$, in the 3D simulation associated with $\alpha$ Centauri A is very close to that of the solar simulation. This is not surprising since the two stars have almost the same effective temperature. Furthermore, as pointed out above, the differences in $F_{\text {kin }}$ between $\alpha$ Centauri A and the Sun are small. As a consequence $\mathcal{R} \propto F_{\text {conv }} / F_{\text {kin }}$ does not differ between $\alpha$ Centauri A and the Sun. This explains why the contribution of the entropy term

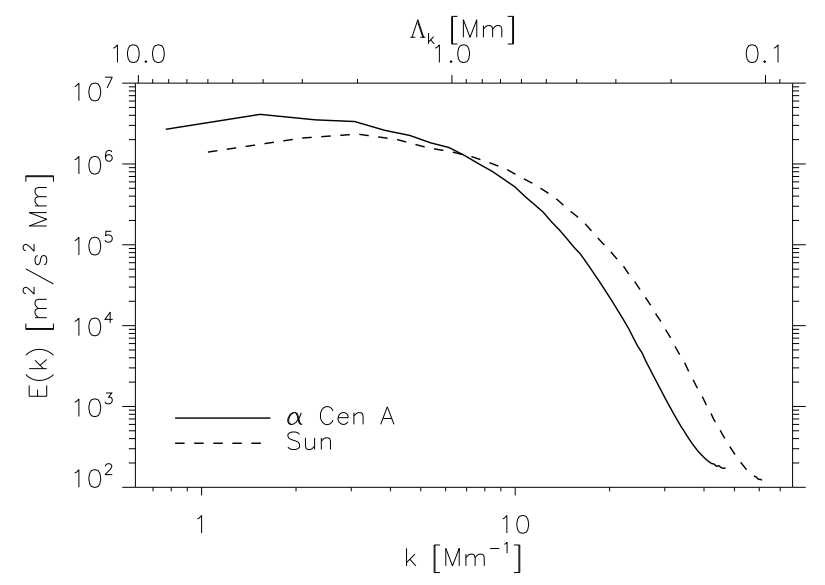

Fig. 6. The kinetic energy spectrum, $E$, as a function of the horizontal wavenumber $k$ (lower axis) and the scale length $\Lambda_{k}=2 \pi / k$ (upper axis) for the layer where $u$ is maximum. The solid line corresponds to the 3D simulation associated with $\alpha$ Centauri A and the dashed line to the one associated with the Sun.

relative to the Reynolds stress is similar between $\alpha$ Centauri A and the Sun.

Characteristic scale length $(\Lambda)$ :

Figure 6 shows the kinetic energy spectrum $E$ as a function of the horizontal wavenumber $k$ and the scale length $\Lambda_{k}=2 \pi / k$ for the layer where $u$ is maximum. As seen in Fig. 6, for the $3 \mathrm{D}$ simulation associated with $\alpha$ Centauri A, $E$ is maximum at a larger scale length compared to the solar simulation. Then, the eddies have a larger characteristic scale length in $\alpha$ Centauri A than in the Sun. This explains why the excitation of p-modes is significantly stronger for $\alpha$ Centauri A than for the Sun.

Since the number of grid points is the same for both simulations, the $\alpha$ Centauri A simulation has a larger physical grid size, thus a smaller maximum wavenumber, and in turn the cut-off in the spectrum occurs at a lower $k$. This explains the earlier dropoff of $E(k)$ for $\alpha$ Centauri A in Fig. 6. For that reason the high wavenumber part (beyond a $k$ value of about $15 \mathrm{Mm}^{-1}$, or a $\Lambda_{k}$ less than $0.4 \mathrm{Mm}$ ) should not be compared directly. On the other hand, the scaling chosen in Fig. 7 allows a direct comparison.

We point out that the characteristic scale length, $\Lambda$, scales as the pressure scale height. Indeed, we have plotted in Fig. 7 the kinetic energy spectrum, $E$, as a function of $k H_{p}$ where $H_{p}$ is the pressure height at the layer where $u$ is maximum. Except at small scale lengths, we see that the $k$-dependency of the spectrum is almost the same between the simulation associated with $\alpha$ Centauri A and the solar one.

The ratio between $H_{p}^{\alpha \operatorname{cen} \mathrm{A}}$ and $H_{p}^{\odot}\left(H_{p}^{\alpha \operatorname{cen} \mathrm{A}} / H_{p}^{\odot} \simeq 1.38\right)$ is very close to the ratio $g_{\odot} / g_{\alpha}$ cen A $(\simeq 1.36)$. This is obviously related to the fact that $H_{p}=P / \rho g \propto T / g$. Accordingly, since $\mathcal{P} \propto \Lambda^{4}$ (see above), we then have $\mathcal{P} / \mathcal{P}_{\odot} \propto\left(\Lambda / \Lambda_{\odot}\right)^{4} \propto$ $\left(H_{p}^{\alpha \operatorname{cen~A}} / H_{p}^{\odot}\right)^{4} \propto\left(g_{\alpha \text { cen A }} / g_{\odot}\right)^{4} \sim 3.4$. For comparison, excitation rates computed for $\alpha$ Centauri A are two times greater than in the Sun.

\subsubsection{Why $\mathcal{P}$ peaks at lower frequency?}

The characteristic eddy turnover time can be estimated as the quantity $\tilde{\tau} \sim L_{h} / u$ where $L_{h}$ is the horizontal extent of the 3D model and $u$ the velocity at a given layer. At the layer where $u$ is maximum, we find that $\tilde{\tau}$, evaluated at the layer where $u$ peaks, is larger in $\alpha$ Centauri A ( $\sim 30 \mathrm{~min})$ than in the Sun $(\sim 23 \mathrm{~min})$. 


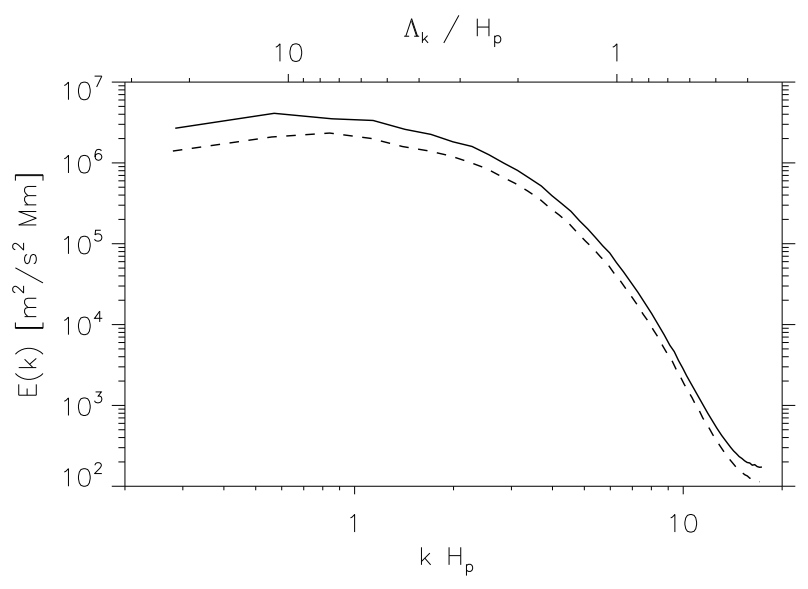

Fig. 7. The kinetic energy spectrum, $E$, as a function of $k H_{p}$ (lower axis) and $\Lambda_{k} / H_{p}$ (upper axis) for the layer where $u$ is maximum. The lines used have the same meaning as in Fig. 6.

This explains that for $\alpha$ Centauri A $\mathcal{P}$ peaks at lower frequency than in the Sun $\left(\omega_{0} \sim 2 \pi / \tilde{\tau}\right.$, cf. Eq. (8)).

Both $u$ and $\Lambda$ are larger for $\alpha$ Centauri A than for the Sun. However, the net result is a larger $\tilde{\tau}$ for $\alpha$ Centauri A.

\subsubsection{Interpretation}

The differences in characteristics of convection between $\alpha$ Centauri A and the Sun can be understood as follows: as seen in Sect. 5.3.1, the characteristic size $\Lambda$ is mainly controlled by $H_{p} \propto T / g$ (for a given composition). The surface gravity for $\alpha$ Centauri A is $\sim 35 \%$ times weaker than for the Sun while the effective temperature is very similar to that of the Sun. Consequently, $\Lambda$ is larger than in the Sun. Furthermore because of the lower gravity, the density at the photosphere is lower than in the Sun. Hence, to transport the same amount of energy per unit surface area by convection, the convective cells must have higher speed $(u)$.

\section{Discussion}

\subsection{Effect of chemical composition}

The star $\alpha$ Centauri A has an iron-to-hydrogen abundance of $[\mathrm{Fe} / \mathrm{H}]=0.2($ see Miglio \& Montalbán 2005). The 3D simulation considered here has a solar abundance. Preliminary work tends to show that, at the given effective temperature, a 3D simulation with a metal abundance 10 times less than the solar one results in mode excitation rates $\sim 2$ times smaller. This can be understood as follows: the radiative flux is larger for a low metallicity than in a medium with a solar metallicity. In that case, to transport the same amount of energy, convection is less vigorous (i.e. lower flux of kinetic energy, $F_{\text {kin }}$ ), leading to a lower efficiency of the driving. If we extrapolate this preliminary result, we can expect that mode excitation rates ought to increase with $[\mathrm{Fe} / \mathrm{H}]$. A quantitative estimate of the expected increase must be performed, however, in particular for $\alpha$ Centauri A, which will require computing a $3 \mathrm{D}$ simulation with a non-solar abundance representative of the surface layers of the star (in progress).

\subsection{Estimation of mode mass}

Mode masses must be evaluated at the layer in the atmosphere where the acoustic modes are predominantly measured. The

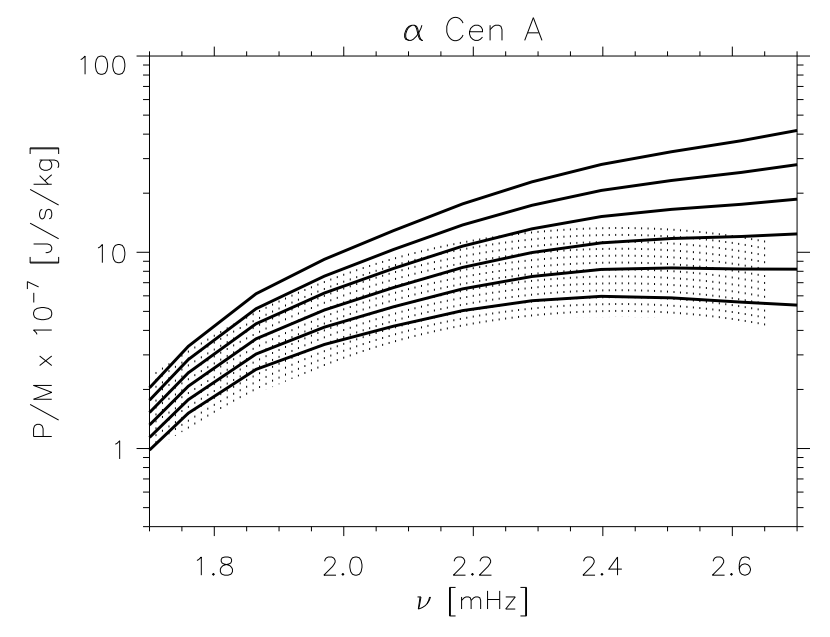

Fig. 8. Ratio of the rates $\mathcal{P}$ at which energy is injected into the p-modes to the mode masses $(\mathcal{M})$ for $\alpha$ Centauri A. The dashed area represents the observed domain for $\mathcal{P} / \mathcal{M}=2 \pi \Gamma\left(v / S_{0}\right)^{2}$ as a function of $v$ (see Sect. 3). The solid lines correspond to the ratio $\mathcal{P} / \mathcal{M}$ where the excitation rates, $\mathcal{P}$, are calculated according to Eq. (1) and the mode masses, $\mathcal{M}$, are evaluated at different heights $h$ above the photosphere. The lower curve corresponds to the photosphere $(h=0)$ and the upper curve to the top of the atmosphere $(h=1000 \mathrm{~km})$. The step in $h$ is $200 \mathrm{~km}$.

result of a comparison with seismic constraints significantly depends on the effective heights $h$ where mode masses are evaluated. Indeed, we plot in Fig. 8 the ratio $\mathcal{P} / \mathcal{M}$ for mode masses evaluated at different heights $h$ in the atmosphere, namely from $h=0$ (the photosphere) up to the top of the simulated domain $(h \simeq 1000 \mathrm{~km})$. This ratio is compared to the quantity $2 \pi \Gamma\left(v / S_{0}\right)^{2}$ obtained from the seismic constraints (Eq. (7)). For $h \gtrsim 600 \mathrm{~km}$ (i.e. for optical depth $\lesssim 0.005$ ), the ratio $\mathcal{P} / \mathcal{M}$ is outside the observational domain.

Seismic observations of $\alpha$ Centauri A were performed using UCLES and UVES spectrographs. UVES and UCLES use a similar technique to measure the acoustic modes (Bedding, private communication). Like other spectrographs dedicated to stellar seismic measurements, the UCLES instrument uses a large number of spectral lines to reach a high enough signal-to-noise ratio. In the case of stellar seismic measurements, it is then more difficult than for helioseismic observations to estimate the effective height $h$ (for the solar case see, e.g., Baudin et al. 2005). A recent work by Kjeldsen et al. (2008) allows us to estimate the value for an effective $h$. Indeed, the authors have found that solar modes measured with the UCLES spectrograph have amplitudes slightly less than those measured by the BiSON network. The instruments of the BiSON network use the potassium (K) spectral line, which is formed at an optical depth $\tau_{500 \mathrm{~nm}} \simeq 0.013$ (see Houdek 2006). The Kjeldsen et al. (2008) results then suggest that acoustic modes measured by UCLES are measured at an effective height $(h)$ slightly below the formation depth of the $\mathrm{K}$ line, i.e. at optical depth slightly above $\tau_{500 \mathrm{~nm}} \simeq 0.013$. Accordingly, we have evaluated the mode masses at that optical depth, which corresponds to $h=470 \mathrm{~km}$.

A more rigorous approach would be to compute an effective mode mass by appropriately weighting the different mode masses associated with the different spectral lines that contribute to the seismic measurement. To infer accurate mode excitation rates from the seismic data of $\alpha$ Centauri A, the mode masses representative of the observation technique and the spectral lines of $\alpha$ Centauri A must be derived. This is, however, beyond the scope of this paper. 


\section{Conclusions}

Theoretical estimations for the energy supplied per unit of time by turbulent convection $(\mathcal{P})$ to $\alpha$ Centauri A acoustic modes were compared to values obtained from observations. This allows us to draw the following conclusions.

\subsection{Differences with the Sun}

Although $\alpha$ Centauri A has an effective temperature very close to that of the Sun, we find here that the $\mathrm{p}$-mode excitation rates $\mathcal{P}$ inferred from the seismic constraints obtained for $\alpha$ Centauri A are about two times higher than in the Sun. These differences are attributed to the fact that the eddies in $\alpha$ Centauri A have a larger characteristic size $(\Lambda)$ than in the Sun. This is related to the weaker surface gravity of $\alpha$ Centauri A.

Furthermore, the p-mode excitation rates for $\alpha$ Centauri A are maximum at lower frequencies than in the Sun. This behavior is related to the eddies having a longer turnover time as a result of a larger $\Lambda$.

The seismic characteristics of the p-modes detected in $\alpha$ Centauri A significantly differ from that of the Sun. They can therefore provide additional constraints on the model of stochastic excitation.

\subsection{Inferred versus modeled excitation rates}

Our modeling gives rise to excitation rates within the error bars associated with the observational constraints. We stress that this modeling was undertaken for $\alpha$ Centauri A independently from the solar case, i.e. without using a formulation fitted on the helioseismic data as is the case, for instance, for the Sun in Chaplin et al. (2005) or in the case for $\alpha$ Centauri A in Houdek (2002). The seismic constraints from $\alpha$ Centauri A then provide a clear validation of the basic underlying physical assumptions included in the theoretical model of stochastic excitation, at least for stars that are not too different from the Sun.

\subsection{Constraints on the description of turbulence: eddy-time correlation}

We find that our theoretical estimations of $\mathcal{P}$, which assume a Lorentzian eddy-time correlation function $\left(\chi_{k}\right)$ and the CMP proposed by Belkacem et al. (2006a), lie in the observed domain. On the other hand, when a Gaussian function is chosen for $\chi_{k}, \mathcal{P}$ is significantly underestimated. The comparison with the seismic data for $\alpha$ Centauri A confirms the results for the solar case obtained by Samadi et al. (2003) that $\chi_{k}$ significantly departs from a Gaussian. As in Samadi et al. (2003), we attribute the departure of $\chi_{k}$ from a Gaussian to diving plumes (i.e. down-flows), which are more turbulent than granules (i.e. the up-flows). This result confirms that a Lorentzian function is a more adequate description for the eddy-time correlation than a Gaussian.

\subsection{Constraints on the modeling of turbulent convection in the equilibrium stellar model}

Calculations involving eigenfunctions computed on the basis of a global 1D model that includes a realistic description of the outer layers of the star (taken from 3D simulations) reproduce much better (see Fig. 2) the seismic data than calculations that use eigenfunctions computed with a standard stellar model built with the MLT and ignoring turbulent pressure. This is because a model that includes turbulent pressure results in higher mode masses $\mathcal{M}$ than a model that ignores turbulent pressure. This can be understood as follows. Within the super-adiabatic region, a model that includes turbulent pressure provides an additional support against gravity, hence has a lower gas pressure and density (see Fig. 1) than a model that does not include turbulent pressure. As a consequence, mode inertia (hence mode masses) are then larger in a model that includes turbulent pressure.

These conclusion are similar to that obtained in the Sun. Indeed, the mode masses considered by Belkacem et al. (2006b) in the case of the Sun were obtained with a 1D model computed using the Gough (1977) non-local mixing-length formulation of convection. The model thus includes turbulent pressure. We do not observe significant differences between excitation rates obtained with this non-local model and those obtained with a "patched" solar computed as described here in the case of $\alpha$ Centauri A. On the other hand, excitation rates computed with mode masses obtained with a "standard" solar model (that is, with no turbulent pressure included) or with a model in which turbulent pressure is included according to the MLT significantly under-estimate the helioseismic constraints.

These results tell that one must compute mode masses from 1D models that include turbulent pressure using a 3D hydrodynamical model or using a non-local description of convection.

\subsection{Need for improved data sets}

As shown by Samadi et al. (2003) in the case of the Sun, contribution of the entropy fluctuations to the excitation cannot be neglected. Furthermore, recently, Belkacem et al. (2006b) have shown that theoretical calculations based on the CMP result in a better agreement with the helioseismic constraints than those based on QNA.

However, in the case of $\alpha$ Centauri A, differences between theoretical calculations that use the CMP and those based on the QNA (see Fig. 3), as well as differences between calculations including driving by entropy fluctuations and those that do not include it (not shown), are of the same order as the observational uncertainties associated with the two data sets. The present seismic constraints therefore are unable to distinguish between these assumptions. This emphasizes the need for more accurate seismic data for $\alpha$ Centauri A.

Acknowledgements. F.K.'s work was possible thanks to a one month grant provided by Observatoire de Paris. We thank T. Bedding for providing us the amplitude spectrum of $\alpha$ Centauri A obtained from the UVES and UCLES spectrographs. We thank Å. Nordlund, R. F. Stein, and R. Trampedach for making their 3D simulation code available. Finally, we thank the referee (M. Steffen) for his useful remarks.

\section{References}

Baudin, F., Samadi, R., Goupil, M.-J., et al. 2005, A\&A, 433, 349

Bedding, T. R., Kjeldsen, H., Butler, R. P., et al. 2004, ApJ, 614, 380

Belkacem, K., Samadi, R., Goupil, M. J., \& Kupka, F. 2006a, A\&A, 460, 173

Belkacem, K., Samadi, R., Goupil, M. J., Kupka, F., \& Baudin, F. 2006b, A\&A, 460,183

Böhm-Vitense, E. 1958, Z. Astrophys., 46, 108

Butler, R. P., Bedding, T. R., Kjeldsen, H., et al. 2004, ApJ, 600, L75

Chaplin, W. J., Houdek, G., Elsworth, Y., et al. 2005, MNRAS, 360, 859

Christensen-Dalsgaard, J., \& Berthomieu, G. 1991, Theory of solar oscillations,

Solar interior and atmosphere (A92-36201 14-92) (Tucson, AZ: University of Arizona Press), 401

Fletcher, S. T., Chaplin, W. J., Elsworth, Y., Schou, J., \& Buzasi, D. 2006, MNRAS, 824

Goldreich, P., Murray, N., \& Kumar, P. 1994, ApJ, 424, 466

Gough, D. O. 1977, ApJ, 214, 196

Grevesse, N., \& Noels, A. 1993, in Origin and Evolution of the Elements, ed. N. Prantzos, E. Vangioni-Flam, \& M. Cassé (Cambridge University Press), 15 
Houdek, G. 2002, in Radial and Nonradial Pulsationsn as Probes of Stellar Physics, ed. C. Aerts, T. R. Bedding, \& J. Christensen-Dalsgaard, IAU Colloq., 185447, ASP Conf. Ser., 259

Houdek, G. 2006, in Proceedings of SOHO 18/GONG 2006/HELAS I, Beyond the spherical Sun, Published on CDROM, ESA SP, 624, 28.1

Kjeldsen, H., Bedding, T. R., Butler, R. P., et al. 2005, ApJ, 635, 1281

Kjeldsen, H., Bedding, T. R., Arentoft, T., et al. 2008, ApJ, 682, 1370

Michaud, G., \& Proffitt, C. R. 1993, in Inside the Stars, IAU Colloq., 137, ASP Conf. Ser., 40, 246

Miglio, A., \& Montalbán, J. 2005, A\&A, 441, 615

Millionshchikov, M. D. 1941, Doklady Acad. Nauk SSSR, 32, 611

Nordlund, A., \& Stein, R. F. 1999, in Stellar Structure: Theory and Test of Connective Energy Transport, ed. A. Gimenez, E. F. Guinan, \& B. Montesinos, ASP Conf. Ser., 173, 91
Rosenthal, C. S., Christensen-Dalsgaard, J., Nordlund, Å., Stein, R. F., \& Trampedach, R. 1999, A\&A, 351, 689

Samadi, R., Belkacem, K., Goupil, M.-J., Kupka, F., \& Dupret, M.-A. 2007a, in ed. F. Kupka, I. Roxburgh, \& K. Chan, IAU Symp., 239, 349

Samadi, R., Georgobiani, D., Trampedach, R., et al. 2007b, A\&A, 463, 297

Samadi, R., \& Goupil, M. 2001, A\&A, 370, 136

Samadi, R., Goupil, M.-J., Alecian, E., et al. 2005, A\&A, 26, 171

Samadi, R., Kupka, F., Goupil, M. J., Lebreton, Y., \& van’t Veer-Menneret, C. 2006, A\&A, 445, 233

Samadi, R., Nordlund, Å., Stein, R. F., Goupil, M. J., \& Roxburgh, I. 2003, A\&A, 404, 1129

Stein, R. F., \& Nordlund, A. 1998, ApJ, 499, 914

Stein, R. F., \& Nordlund, Å. 2001, ApJ, 546, 585

Trampedach, R. 1997, Master's thesis, Aarhus University 